\title{
Politique et humanitaire à l'échelle locale pendant la guerre d'Algérie : jumelages et colonies de vacances dans l'Ouest de la France
}

Local politics and humanitarian work during the Algerian War: twinnings and holiday camps in western France

\section{Yves Denéchère}

\section{OpenEdition \\ Journals}

Édition électronique

URL : http://journals.openedition.org/abpo/3672

DOI : $10.4000 / a b p o .3672$

ISBN : 978-2-7535-6497-8

ISSN : 2108-6443

Éditeur

Presses universitaires de Rennes

Édition imprimée

Date de publication : 30 juin 2017

Pagination : 145-169

ISBN : 978-2-7535-6495-4

ISSN : 0399-0826

Référence électronique

Yves Denéchère, "Politique et humanitaire à l'échelle locale pendant la guerre d'Algérie : jumelages et colonies de vacances dans l'Ouest de la France ", Annales de Bretagne et des Pays de l'Ouest [En ligne], 124-2 | 2017, mis en ligne le 17 juillet 2019, consulté le 02 janvier 2020. URL : http:// journals.openedition.org/abpo/3672; DOI : 10.4000/abpo.3672 


\title{
Politique et humanitaire à l'échelle locale pendant la guerre d'Algérie : jumelages et colonies de vacances dans l'Ouest de la France
}

\author{
Yves DENÉCHÈRE \\ Professeur d'histoire contemporaine, Université d'Angers, CERHIO CNRS
}

Dix ans après l'ouvrage collectif dirigé par Raphaëlle Branche et Sylvie Thénault sur les expériences métropolitaines de la guerre d'Algérie ${ }^{1}$, des pistes de recherche visant à mieux connaître les vécus et les ressentis des populations ont été suivies par les historiens, pas si nombreux encore, qui travaillent sur cet objet. Développer la problématique de la rencontre entre les sociétés vivant de part et d'autre de la Méditerranée durant le conflit demeure une nécessité afin de reconstruire un passé difficile à appréhender, ne serait-ce qu'au niveau des termes utilisés pour simplement qualifier les populations vivant en Algérie : "Français musulmans ", " musulmans ", " Algériens ${ }^{2}$ ». Cette approche est indispensable pour tenter de produire un récit historique équilibré, prenant en compte les expériences individuelles des différents acteurs, combattants et civils, femmes et hommes, adultes et enfants.

À l'échelle de l'Ouest de la France, quelques dizaines de mémoires de maîtrise ou de master ont été produits depuis les années 1980 dans les départements d'histoire et les centres de recherche des universités. Les plus anciens de ces travaux ont été réalisés lorsque l'accès aux archives était encore très restreint et la plupart d'entre eux ont donc porté sur la manière dont la presse régionale et locale a traité de la guerre d'Algérie.

1. BRANCHE, Raphaëlle, THÉNAULT, Sylvie (dir.), La France en guerre 1954-1962. Expériences métropolitaines de la guerre d'indépendance algérienne, Paris, Autrement, 2008.

2. Sur la difficulté de l'utilisation des termes et le choix entre le vocabulaire colonial ou les mots d'aujourd'hui, voir STORA, Benjamin, Les mots de la guerre d'Algérie, Toulouse, Presses universitaires du Mirail, 2005; BRANCHE, Raphaëlle, La guerre d'Algérie : une histoire apaisée? Mémoires et débats, Paris, Seuil, 2005, "Que faire de mots de l'époque coloniale? ", p. 349-359; KADRI, Aïssa (dir.), Instituteurs et enseignants en Algérie 1945-1975. Histoire et mémoires, Paris, Karthala, 2014, "Les mots de la colonisation ", p. 15-18. 
À partir des années 1990, sont venus s'y adjoindre des mémoires consacrés à l'impact des " événements d'Algérie " sur la politique locale ou l'immigration. Plus récemment, les problématiques se sont diversifiées en phase avec le renouvellement historiographique et ont été travaillées les questions du deuil des soldats français tués en Algérie ou des mémoires des appelés ${ }^{3}$. Il s'agit de mieux comprendre comment la société a été touchée par cette guerre, à l'instar de l'interrogation de Jean-Luc Marais : " Que pensait la masse des Angevins ${ }^{4}$ ? " En élargissant cette question à un espace plus vaste, il reste beaucoup à faire pour mieux cerner ce que les populations de l'Ouest ont eu à connaître, ont pensé de la guerre d'Algérie, comment elles se sont engagées ou pas sur cette question si complexe. Et aussi quelles représentations pouvaient-elles se faire des populations vivant en Algérie, notamment à travers la propagande mise en œuvre par les autorités pour créer et valoriser des connexions entre les sociétés de métropole et d'Algérie. Parmi les opérations de rapprochement décidées au plus haut niveau de l'État et mises en pratique à l'échelle locale, les parrainages entre villes et les jumelages entre départements métropolitains et arrondissements d'Algérie ont été très peu étudiés, sans doute parce qu'ils ont été complètement oubliés, à l'inverse des premiers jumelages européens qui leur sont contemporains ${ }^{5}$. Comme ceux-ci, ceux-là ont pourtant été l'occasion de contacts, de rencontres et d'actions humanistes et humanitaires, entre Français métropolitains et populations d'Algérie, notamment dans le cadre de colonies de vacances organisées en France ${ }^{6}$. Mais le dépouillement bibliographique révèle seulement quelques contributions portant sur les parrainages et les jumelages pendant la guerre d'Algérie, chacune étudiée sous un angle particulier?

3. D'après la consultation de la base de données bibliographique THEO, réalisée par le CERHIO (Centre de Recherches Historiques de l'Ouest) : [http://services.univ-rennes2. $\mathrm{fr} /$ memorable/interro/accueil.php?act $=a c c \& b=2]$.

4. MARAIS, Jean-Luc, Le Maine-et-Loire aux XIXe et XXe siècles. Histoire de l'Anjou, tome 4, Paris, Picard, 2009, p. 252.

5. Les travaux sur l'histoire des jumelages se concentrent sur les jumelages européens et n'évoquent pas ceux de la guerre d'Algérie. Voir ChOMBARD-Gaudin, Cécile, " Pour une histoire des villes et communes jumelées ", Vingtième Siècle, Revue d'Histoire, 1992/3, $\mathrm{n}^{\circ} 35$, p. 60-77; VION, Antoine, "L'invention de la tradition des jumelages : mobilisations pour un droit (1951-1956) ", Revue Française de Science Politique, 2003/4, n 53, p. 559-582; DEFRANCE, Corinne, "Les premiers jumelages franco-allemands 1950-1963 ", Lendemains, $1996, \mathrm{n}^{\circ} 84$, p. 83-95 et "Les jumelages franco-allemands : aspects d'une coopération transnationale ", Vingtième Siècle, Revue d'Histoire, 2008, n 99, p. 189-201.

6. Ces colonies de vacances pour enfants d'Algérie en France ne sont pas étudiées dans Downs, Laura Lee, Childhood in the Promised Land: Working-Class Movements and the Colonies de vacances in France de 1880 à 1960, Duke University Press, 2002, trad. française : Histoire des colonies de vacances, de 1880 à nos jours, Paris, Perrin.

7. Les deux plus solides sont : BouRDIN, Gérard, « Les ambiguïtés d'un rapprochement : parrainages et jumelages, 1956-1962 ", dans BRANCHE, Raphaëlle, THÉNAult, Sylvie (dir.), La France en guerre..., op. cit., p. 82-100, recherche réalisée surtout à partir d'archives départementales en s'appuyant sur le réseau des correspondants de l'IHTP; HARDT, Lucas, «Ferienlager gegen die Rebellion. Jumelages und colonies de vacances im Algerienkrieg 
La documentation mobilisée pour cet article montre pourtant que, tant au niveau national que local, les sources sont diverses, d'une grande richesse et permettent de faire l'histoire de ces jumelages qui ont laissé si peu de mémoire. Les archives départementales (surtout dans les versements des préfectures et des conseils généraux, de la série W) et les archives municipales recèlent des traces non négligeables de ces relations, le plus souvent à la surprise de leurs conservateurs. La presse locale, par exotisme parfois, par conviction et parti pris le plus souvent, est prompte à relayer la propagande qui entoure les rapports avec l'Algérie. Lors d'entretiens, sources orales indispensables pour faire une histoire du temps présent, l'exhumation d'archives privées (photos, correspondances) vient compléter le matériel local à la disposition de l'historien. Les grands centres nationaux d'archives constituent des ressources essentielles pour replacer les actes dans le contexte politique car les jumelages comme les colonies de vacances font l'objet d'une organisation, d'un suivi et de bilans qui s'inscrivent dans le cadre de la guerre psychologique menée en Algérie. Les archives du ministère des Affaires étrangères (fonds du SGCA : secrétariat d'État aux Affaires algériennes), les Archives nationales (fonds du ministère de l'Intérieur) et les Archives nationales d'Outre-Mer (ANOM - Aix-en-Provence) conservent les sources essentielles, sans oublier le Service historique de la défense (SHD - Vincennes).

Dans les sources il apparaît clairement que les parrainages de villes, spontanés ou encouragés, ont eu peu d'échos dans l'Ouest. En revanche, les départements ont suivi, bon gré mal gré, le mouvement général des jumelages avec des arrondissements algériens. Dans ce cadre, les actions humanitaires en faveur des enfants, notamment les colonies de vacances, ont constitué une " action phare ", parce que propice à la mobilisation humaniste des métropolitains et des structures associatives et religieuses si nombreuses dans l'Ouest.

\section{De la variabilité des engagements dans les parrainages puis dans les jumelages}

\section{Peu de parrainages de villes d'Algérie dans l'Ouest}

Au printemps 1956, le Conseil des communes d'Europe (CCE) - qui a initié des jumelages franco-allemands -, le Mouvement national des élus locaux (MNEL), le Centre national des indépendants et paysans (CNIP), mais aussi l'Union pour le salut et le renouveau de l'Algérie française, que vient de créer Jacques Soustelle, s'associent pour lancer les parrainages de villes ${ }^{8}$. En juin, une proposition de résolution est déposée à l'Assemblée nationale, invitant " le gouvernement à prendre toutes mesures afin

(1959-1962) ", Journal of Modern European story, vol. 11, 2013/3, p. 361-374, recherche réalisée surtout à partir des archives du ministère des Affaires étrangères.

8. BouRDin, Gérard, "Les ambiguïtés d'un rapprochement... ", op. cit., p. 82-83. Jacques Soustelle a été Gouverneur général de l'Algérie de janvier 1955 à janvier 1956. 
de développer la campagne de solidarité Métropole-Algérie en favorisant et généralisant les jumelages entre villes et villages de la métropole et ceux d'Algérie $^{9}$ ». Mais les initiatives sont plutôt locales, relevant de la volonté individuelle d'élus, de relations personnelles, de Français d'Algérie installés dans les villes françaises. En 1957, le MNEL publie un livret consacré $\mathrm{au}$ " Parrainage de communes algériennes par des villes de la métropole ", présenté comme une réponse à toutes les manœuvres de déstabilisation de l'Algérie opérées par des communistes ou des nationalistes. Le mouvement établit une liste de 150 parrainages, d'autres sources en indiquent environ 130. Parmi eux, on relève seulement dans l'Ouest de la France : Brest et Mostaganem, Lisieux et Guyotville, Quimper et Affreville, Château-du-Loir et Thiersville, Chinon/Loches et Collo. Nantes a refusé par deux fois le parrainage d'Orléansville proposé par le MNEL ${ }^{10}$.

Le maire de Thiersville passe à Château-du-Loir en octobre 1956 et lance un appel à la solidarité en faveur des enfants algériens. D'un côté comme de l'autre, de grandes manifestations de fraternité et de grandes ambitions sont professées ${ }^{11}$. Située en Oranie, la ville a souhaité nouer des liens avec la Sarthe " en mémoire de la compagnie de CRS du Mans qui y avait stationné et laissé de bons souvenirs ". En avril 1958, le maire et son épouse sont assassinés, ce qui provoque une grande émotion à Châteaudu-Loir ${ }^{12}$. Le conseil municipal d'Écommoy, dont le maire est un ami de Jacques Soustelle, constatant que les communes en Algérie sont bien plus étendues que celles de la métropole, propose que ces jumelages se fassent par groupes de communes sarthoises ${ }^{13}$.

Les parrainages entre villes sont relancés par le plan de Constantine, l'objectif étant fixé à 1500, ce qui implique un décuplement du phénomène. Après le référendum du 28 septembre 1958 sur la constitution de la Ve République, approuvé à 82,6\% en France et à 96 \% en Algérie, un plan de cinq ans est annoncé à Constantine par de Gaulle le 3 octobre 1958. Il vise à construire une "Algérie nouvelle " et comporte des mesures de mise en valeur des terres, la construction de logements et porte une grande attention à l'enfance considérée comme un enjeu essentiel pour l'avenir, d'où le développement d'une scolarisation massive de rattrapage ${ }^{14}$. Dans ce grand mouvement, Saint-Fiacre-sur-Maine et Saint-Brévin-les-Pins

9. Arch. nat., F1 cII 546, proposition de résolution n ${ }^{\circ} 2328$, procès-verbal de séance de l'Assemblée nationale, 26 juin 1956.

10. Arch. nat., F1 cII 546, MNEL, " Parrainage de communes algériennes par des villes de la métropole ", s. d., [1957]; Archives municipales de Nantes, 1144 W 108, notes des 2 octobre 1956 et 4 octobre 1957.

11. Arch. dép. de la Sarthe, 1359 W 137, compte rendu des renseignements généraux, 30 octobre 1956.

12. BouRdiN, Gérard, "Les ambiguïtés d'un rapprochement... ", art. cit. dans BrANCHE, Raphaëlle, ThÉNAult, Sylvie (dir.), La France en guerre..., op. cit., p. 84 et 86.

13. Arch. dép. de la Sarthe, 1359 W 137, délibération du conseil municipal d'Écommoy, 30 juin 1956.

14. Une présentation du plan à l'époque dans CotTA, Alain, «Les perspectives décennales du développement économique de l'Algérie et le plan de Constantine ", Revue 
(Loire-Atlantique) se jumellent avec deux villes d'Oranie, respectivement Damesme et La Calle. Pour la municipalité de Saint-Fiacre, qui compte 600 habitants, " c'est un honneur de répondre positivement à la demande du maire de Damesme "... dont on ne connaît pas les motivations. Une cérémonie officielle, avec défilé et banquet, a lieu le 17 juillet 1959. Dans une correspondance de novembre 1959, on peut lire que La Baule et Pornic ont également accepté de " parrainer les villes algériennes " côtières de Arzew et Port-aux-Poules, elles aussi situées en Oranie, ce qui peut laisser penser à un plan d'ensemble ${ }^{15}$.

Mais tous les parrainages ne sont pas actifs. Certains n'existent même pas dans les faits. Par exemple, sur une liste figurent les parrainages de Nantes et Boghni (département de Tizi-Ouzou) et d'Angers et Ben-Chicao (département de Médéa) mais les recherches entreprises prouvent qu'il ne s'agissait que de projections, ces deux parrainages n'ont jamais été signés, ni même étudiés par les conseils municipaux ${ }^{16}$. Quant aux parrainages effectifs, ils génèrent peu d'activités, restées surtout symboliques. Les " villes-marraines " organisent quelques actions de solidarité envers les "villes-filleules", ce sont donc clairement des relations déséquilibrées ${ }^{17}$. Ces parrainages sont donc de plus en plus souvent qualifiés de "jumelages ", terme apparaissant comme moins condescendant et impliquant l'idée d'échanges dans les deux sens. Quoi qu'il en soit, le mouvement marque le pas en 1959, d'où une réorientation par les autorités vers les jumelages de départements métropolitains avec des arrondissements d'Algérie.

\section{Des adhésions critiques aux injonctions des jumelages venues d'en haut}

Au début du mois de mai 1959, tous les préfets reçoivent une circulaire du Premier ministre Michel Debré lançant la grande opération de jumelage des départements métropolitains avec les arrondissements d'Algérie, avec un objectif ambitieux : "Permettre aux Français vivant sur les deux rives de la Méditerranée de mieux se connaître et de s'entraider. " Peut-être que l'influence de Jacques Soustelle, qui avait encouragé les parrainages de villes, n'est pas étrangère à cette orientation, puisqu'il est alors ministre délégué auprès du Premier ministre, chargé du Sahara, des territoires d'outre-mer

Économique, vol. 10, nº 6, 1959, p. 913-946; Ordonnance $\mathrm{n}^{\circ}$ 58-759 du 20 août 1958 relative au plan de scolarisation et d'éducation de base en Algérie.

15. Arch. dép. de Loire-Atlantique, 270 W 335, délibérations du conseil municipal de Saint-Fiacre-sur-Maine, 8 mai 1959; lettre du maire au préfet, 4 juillet 1959; lettre d'un conseiller technique du 27 novembre 1959.

16. Arch. nat. d'Outre-mer, $1 \mathrm{~K}$ 1288, liste des parrainages de communes, 2 mai 1959. Il n'y a pas la moindre trace de ces parrainages dans les archives municipales de Nantes et d'Angers.

17. Cela est bien montré dans ANDRÉ, Marc, "Quand Lyon parrainait Oran (1956-1962)", Bulletin Municipal Officiel de la ville de Lyon, mars 2012. 
et des départements d'outre-mer. Dix jours plus tard, suit une liste proposant les jumelages pressentis " en vue de développer et de créer des liens concrets de solidarité sur le plan humain ". L'opération a été préparée en amont en un temps très court à partir de février 1959 et à l'initiative du SGAA, pour répondre à la nécessité de développer et de coordonner toutes les actions de parrainage. Plusieurs ministres sont appelés à mobiliser leurs administrations pour faire vivre ces jumelages en y intégrant les activités de solidarité envers les populations d'Algérie : ministères des Armées, de l'Éducation nationale, des Anciens Combattants et des Victimes de guerre, de la Santé publique et de la Population. Pour l'armée, « il ne s'agit pas de faire de la charité, mais essentiellement de concourir à l'œuvre de promotion humaine engagée en Afrique du Nord "... et de le faire savoir ${ }^{18}$. Il apparaît clairement qu'il s'agit là d'une des formes de la guerre psychologique menée en Algérie.

Pour le Gouvernement général en Algérie, la dimension matérielle des jumelages est importante (envois de dons, financement de constructions, etc.), mais l'essentiel est de "multiplier les relations entre métropolitains et algériens " et " la promotion humaine des populations " : formation professionnelle, bourses d'enseignement, bourses artistiques, aide technique, échanges (colonies, correspondances...). Toutes les relations déjà existantes, qu'elles relèvent d'associations ou de communes, sont invitées à intégrer le cadre de jumelage département/arrondissement ${ }^{19}$.

Les conseils généraux sont saisis du projet du gouvernement lors de leur première session de l'année 1959, au printemps. La pression est forte pour obtenir très vite leur adhésion sans que puissent se développer des débats sur la situation en Algérie. Le Morbihan est l'un des premiers à prendre une décision positive, le 19 mai, suivi de l'Ille-et-Vilaine le lendemain; tous les deux se jumellent avec l'arrondissement de Tablat (département de Médéa). Le 20 mai également, la Loire-Atlantique et la Vendée se jumellent avec Paul-Cazelles (également du département de Médéa). Puis la Loire-Atlantique décide d'assumer seule le jumelage et la Vendée choisit l'arrondissement de Khenchela (département de Batna ou de l'Aurès) ${ }^{20}$.

La réactivité des départements de la Mayenne et de la Sarthe est également vive. Dès leurs séances respectives des 22 et 23 mai 1959, ils s'engagent dans le jumelage avec l'arrondissement de Kerrata (département de Sétif). Au Mans, la discussion est cependant rude. Les opposants développent trois arguments : le caractère systématique de ces jumelages quasi imposés, la dimension politique qu'ils comportent, l’inefficacité du

18. Serv. Hist. de la Défense, 1 H 1133, dossier de travail sur les jumelages des départements; 1 H 2462, étude de l'État-major sur "Les parrainages Métropole-Algérie ", 22 janvier 1959; note " situation des parrainages au 10 décembre 1959 ".

19. Serv. Hist. de la Défense, 1 H 2462, lettre du délégué général du gouvernement en Algérie, 30 juin 1959.

20. Arch. dép. de Loire-Atlantique, 2070 W 335, télégramme du préfet, 22 mai 1959; La dépêche Quotidienne d'Algérie, 9 décembre 1959. 


\section{Figure 1 - Localisation des arrondissements d'Algérie jumelés avec des départements de l'Ouest (1959-1962) \\ (Cartographie inédite; source : Arch. nat., F1 cII 546)}

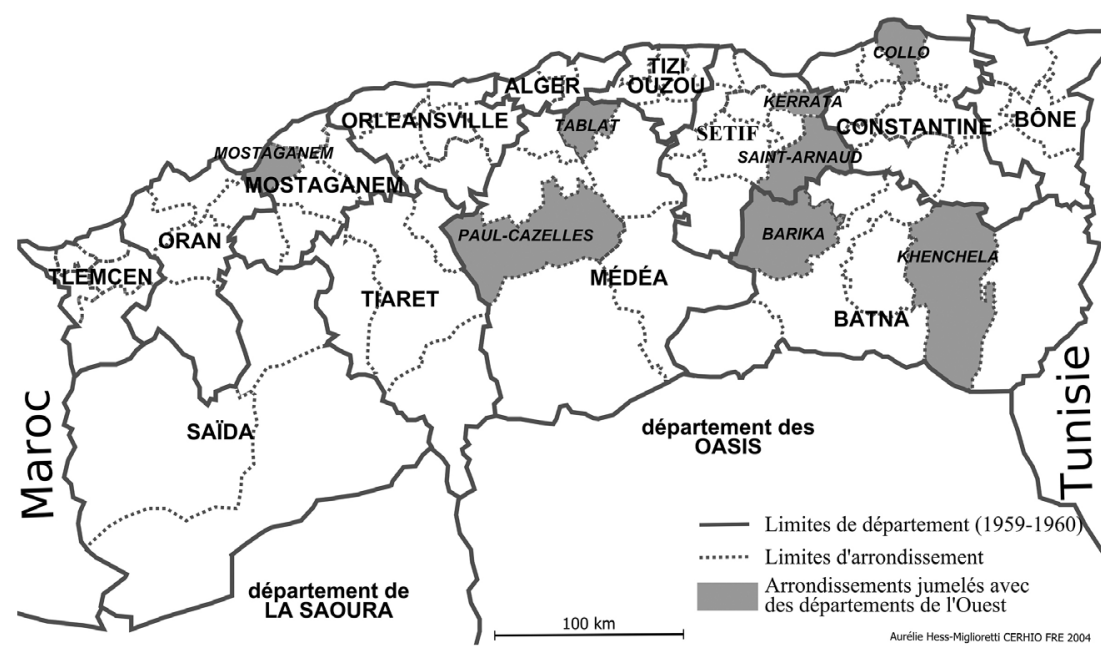

système d'aide. Un débat s'engage sur l'opportunité d'appeler cette opération " jumelage ", qui implique normalement des relations avec une autre nation... Le jumelage de La Flèche après la Deuxième Guerre mondiale avec des villes de Normandie détruites est rappelé, mais on le nommait " parrainage "... Considérant que l'Algérie " ne peut être considérée comme une terre étrangère ", l'ancien ministre socialiste Christian Pineau, se demande si l'on n'a pas voulu " nous demander de prendre ainsi position sur une formule d'intégration ". Pour sa part, le conseiller général communiste Robert Manceau s'interroge sur le sens caché de l'opération : "Je ne vois pas très bien comment vous pourrez convaincre les Algériens qu'ils descendent eux aussi des Gaulois. " Lors du vote, le jumelage est acquis par 13 voix pour, 7 contre et 6 abstentions. Une délégation importante des deux départements, de 13 personnes, dont les deux préfets, les deux présidents des conseils généraux et Robert Buron, conseiller général et ministre des Travaux publics, des Transports et du Tourisme, se rend à Kerrata dès le début octobre 1959. La presse se fait l'écho des " conditions de vie bouleversantes " que décrit le président du conseil général de la Sarthe donnant d'emblée au jumelage une dimension humanitaire : "Il y a là-bas des femmes, des enfants et des vieillards, qui, dans leur misère mais aussi dans leur âme, attendent de vous un geste de solidarité humaine. " La presse d'Algérie rend également compte de la visite ${ }^{21}$. Dans la foulée (30 octobre 1959), Laval décide de se jumeler avec Périgotville, une des villes de l'arron-

21. Arch. dép. de la Sarthe, 1259 W 137, le préfet au ministre de l'Intérieur, 25 mai 1959; compte rendu des renseignements généraux, 25 mai 1959; "Le Conseil général de 
dissement, en vue de " liens sentimentaux, matériels aussi ». D’autres villes du département suivent : Mayenne avec Tizi N'Bechar; Château-Gontier avec Chevreul $^{22}$.

Dans les Deux-Sèvres, la décision de se jumeler avec l'arrondissement de Collo (département de Constantine) est prise dès le printemps, après un débat animé au cours duquel plusieurs élus critiquent le caractère imposé de l'opération. Ils estiment que les habitants de Collo (dont le chef-lieu est parrainé par Chinon et Loches) ne sont pas demandeurs, ou du moins qu'on ne leur a pas demandé leur avis. Le conseil général décide donc d'orienter son aide vers les camps de regroupement forcé de populations ${ }^{23}$. Ce qui constitue en quelque sorte un détournement de l'opération. La misère des populations civiles déplacées de force - environ deux millions de personnes - est alors dénoncée par des associations comme la Cimade du pasteur Jacques Beaumont ou Terre des Hommes créée en 1960 par Edmond Kaiser en réaction à la situation des enfants dans les camps ${ }^{24}$. En choisissant d'aider spécifiquement ces populations, les Deux-Sèvres et d'autres départements affirment un positionnement critique vis-à-vis des jumelages et sur la manière dont est menée la guerre en Algérie.

Fin août 1959, 54 jumelages entre départements métropolitains et arrondissements algériens ou sahariens ont été déjà formellement acceptés, dont dans l'Ouest : Deux-Sèvres/Collo, Calvados/Cherchell, Finistère/ Mostaganem, Ille-et-Vilaine et Morbihan/Tablat, Loire-Atlantique/Paul Cazelles, Mayenne et Sarthe/Kerrata, Vendée/Khenchela. L'Orne et l'Indreet-Loire sont cités en exemple, pour la mise en pratique rapide de l'opération $^{25}$.

\section{Deux départements moins réactifs}

À la fin de décembre 1959 on compte 64 jumelages officiels, d'autres suivent encore. Finalement, 14 départements refusent tout jumelage. Le ministère de l'Intérieur demande à connaître « quelles sont les raisons du

la Sarthe a siégé ", Ouest-France, 25 mai 1959; Ouest-France et La Nouvelle République, 20 octobre 1959; La dépêche de Constantine et de l'Est algérien, 6 et 9 octobre 1959.

22. Foucault, Rémy, " Mayenne-Kerrata (1959-1962), un jumelage franco-algérien ", L'Oribus, $\mathrm{n}^{\circ} 86$, janvier 2013, p. 9-22, illustré avec des photographies d'articles de la presse locale et avec la retranscription du rapport du voyage de la délégation sarthoise et mayennaise à Kerrata en 1959.

23. Arch. dép. des Deux-Sèvres, 1206 W 1, procès-verbal de la séance du 12 janvier 1960, rappelant les termes du débat de la session précédente.

24. KAISER, Edmond, La marche aux enfants, Lausanne, Éditions Pierre-Marcel Favre, 1979, p. 209-225, reproduisant l'article de Jacques Beaumont "Réfugiés en Algérie ", $L a$ Réforme, no 765, 14 novembre 1959. Situation également dénoncée dans RoCARD, Michel, Rapport sur les camps de regroupement forcé, 1959, édition critique en 2003, Paris, Mille et une nuits.

25. Arch. du ministère des Affaires étrangères, SGAA, "Fiche d'information n ${ }^{\circ} 1$ sur les jumelages entre départements métropolitains et arrondissements algériens ", 10 septembre 1959; « Fiche d'information n 2 ", 25 octobre 1959. 


\section{Figure 2 - Jumelages entre départements métropolitains et arrondissements d'Algérie (1959-1962)}

(Cartographie inédite; source : Arch. nat., F1 cII 546)

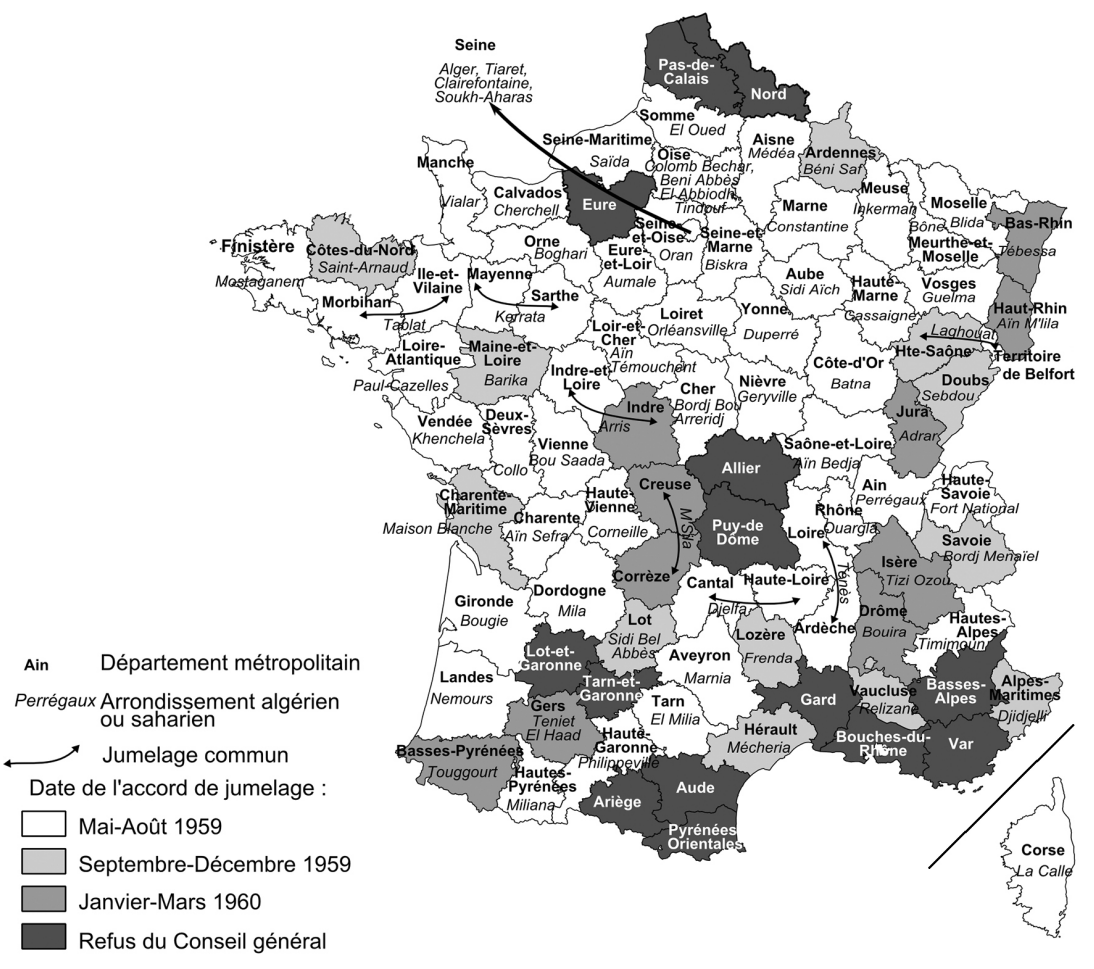

Aurélie Hess-Miglioretti CERHIO (UMR6258)

refus $^{26}$ ". Elles sont toujours politiques. Ainsi le conseil général du Nord réaffirme sa solidarité fraternelle avec ceux qui souffrent de la guerre, mais estime qu'il s'agit là d'une opération imposée d'en haut et pose la question des garanties " en ce qui concerne la pleine liberté de choix et le caractère démocratique de l'élection des représentants des populations musulmanes et européennes avec lesquelles il entrerait en contact ". Le refus définitif est confirmé en janvier 1960. Comme certains départements français, des arrondissements d'Algérie s'opposent à ces jumelages venus d'en haut ${ }^{27}$.

26. Arch. nat., F1 cII 546, SGAA, fiche d'information n 3, 20 décembre 1959; liste des jumelages arrêtée au 22 décembre 1959; lettre circulaire du Premier ministre aux préfets, 6 mai 1959; liste des propositions de jumelage, 16 mai 1959; télégramme du 22 mai 1959.

27. Arch. nat., F1 cII 546, motion votée par le conseil général du Nord, s.d.; carte et liste des arrondissements algériens jumelés, fin 1960. 
Dans l'Ouest, il n'y a aucun refus mais deux départements sont en retard sur le plan de marche du gouvernement. Le département des Côtes-duNord " est réticent " et renvoie son vote. René Pleven, président du Conseil général depuis 1948, est favorable à la politique algérienne du général de Gaulle, « le Français le plus capable de ramener la paix en Algérie et d'éviter la séparation de l'Algérie et de la France ", mais il demande sans cesse à de Gaulle de préciser son calendrier de l'autodétermination ${ }^{28}$. Finalement, en décembre 1959, le département accepte de se jumeler avec l'arrondissement de Saint-Arnaud (département de Sétif). Au cours du long débat qui a précédé le vote, avec l'opposition des élus communistes et socialistes, le conseil général décide que l'aide doit aller prioritairement aux populations des " centres d'hébergement " - en fait les camps de regroupement - en se référant à la presse qui fait état de leurs conditions de vie misérables, du Canard Enchaîné à France-Soir ${ }^{29}$. Comme dans les Deux-Sèvres, cette réorientation de l'aide permet aux élus d'accepter le jumelage sans cautionner le caractère politique de l'opération.

En mai 1959, le gouvernement propose au département de Maine-etLoire un jumelage avec l'arrondissement de Barika (département de Batna) situé à environ $170 \mathrm{~km}$ de Sétif et qualifié de "région déshéritée tant sur le plan économique que social et administratif ". Les 82000 habitants sont très dispersés avec seulement 200 Européens, presque tous fonctionnaires. Barika, chef-lieu d'arrondissement, compte 6000 habitants. Sur le plan politique, la population a basculé en 1956 du côté du FLN « en raison de la faible présence militaire française ${ }^{30} "$.

Le conseil général examine la proposition lors de sa session de mai mais réserve sa décision. Le préfet répond à l'insistance du ministère de l'Intérieur - qui réclame la position du Maine-et-Loire - qu'une décision de principe a été prise "sous réserve d'obtenir des précisions sur les incidences financières qui en résulteraient ". Mais c'est une manière de présenter les choses sous un jour positif. Alors que deux mois plus tard le préfet lui propose une visite à Barika, le président du conseil général rappelle clairement que sans les indications financières demandées il ne peut prendre de décision. Manifestement réservé, il refuse de se laisser embrigader et justifie sa prudence par le souci des deniers publics. Il faut attendre la session d'automne (17 novembre 1959) pour que l'assemblée se prononce favorablement sur le principe du jumelage... à l'unanimité ${ }^{31}$,

28. Voir Bougeard, Christian, René Pleven. Un Français libre en politique, Rennes, PUR, 1994, chapitre XIV : "Un centriste face à l'installation de la République gaullienne : du soutien à la prise de distance (1958-1962) ", p. 325-350.

29. Arch. dép. des Côtes-d'Armor, 128 W 10, rapport de délibérations du conseil général des Côtes-du-Nord, 7 janvier 1960.

30. Arch. dép. de Maine-et-Loire, 303 W 376, plaquette de présentation de Barika réalisée par Le Courrier de l'Ouest en 1960.

31. Arch. dép. de Maine-et-Loire, 303 W 376, télégramme du préfet au ministre de l'Intérieur, 25 mai 1959; lettre du président du conseil général au préfet, 29 juillet 1959; délibération du conseil général, 17 novembre 1959; " Jumelage de l'Anjou avec un arron- 
alors que d'autres départements se sont jumelés plus tôt mais après bien davantage de débats internes.

La presse locale en rend compte et présente un exemple de jumelage qui fonctionne bien, celui du département voisin d'Indre-et-Loire avec l'arrondissement d'Arris (également du département de Batna) qui, selon un de ses promoteurs, "n'est pas une opération-charité mais une œuvre de collaboration humaine et technique ». Le conseil général d'Indre-et-Loire a déjà organisé deux voyages d'études dans "son " arrondissement algérien en mai et octobre; en retour, des maires algériens ont été accueillis dans des communes. Selon la presse, "c'est à un travail analogue, d'une portée humaine considérable que le Conseil général de Maine-et-Loire a été invité à donner son assentiment ${ }^{32}$ ".

Le ministère de l'Intérieur rappelle à l'ordre les départements qui ont pris du retard dans l'organisation des visites sur place jugées comme des préalables indispensables à toute action, l'information de l'opinion publique étant une nécessité absolue. La délégation du conseil général du Maine-et-Loire qui se rend à Barika en mai 1960 n'est pas menée par son président, indisponible. Elle se compose du secrétaire général de la préfecture et de trois conseillers généraux, tous étiquetés " divers droite ". Rien à voir donc avec l'imposante délégation de Mayenne et de Sarthe à Kerrata, qui visite les localités les plus importantes, des écoles, des SAS (sections administratives spécialisées de l'armée, chargées de promouvoir l'œuvre sociale de la France), rencontre des anciens combattants, des autorités musulmanes ${ }^{33}$...

Le rapport de fin de mission signale que «malgré toutes ces pressions [celles du FLN] la population musulmane souhaite la présence française ", que la "pacification est loin d'être achevée ". La visite confirme une " région fort mal exploitée " : l'eau est mal utilisée, les connaissances agricoles insuffisantes, facteurs auxquels s'ajoute " peut-être une nonchalance endémique, due au climat, aux coutumes et aussi au manque de travail ". L'hôpital manque de lits, les écoles de fournitures, les centres pour nourrissons de vêtements... Les projets envisagés sont de plusieurs ordres et semblables à ceux déjà mis en place par les départements voisins : visite d'une délégation de Barika en Anjou, organisation de colonies de vacances, formation de cadres agricoles par des stages, jumelage de la Croix-Rouge angevine avec celle de Barika. La presse relaie le sentiment très positif de la délégation au terme d'un " voyage très constructif et apaisant ", soulignant " un accueil

dissement algérien (celui de Barika dans le Sud-Constantinois) ", " L'exemple de l'Indreet-Loire ", Le Courrier de l'Ouest, 17 novembre 1959.

32. "Le Conseil général a adopté le principe du jumelage du département avec l'arrondissement de Barika ", "Faisons connaissance avec Barika, notre arrondissement jumeau ", Le Courrier de l'Ouest, 18 novembre 1959.

33. Arch. dép. de Maine-et-Loire, 303 W 376, SGAA, fiche d'information no 4, 26 mars 1960, établie suite à une réunion du 18 février 1960; dossier d'organisation de la visite à Barika, programmes, coupures de presse. 
enthousiaste des populations " car " les Algériens veulent être - quelle que soit la forme politique qui les régira - Français avant tout ${ }^{34}$ ". De la lecture des comptes rendus, se dégage l'impression d'un certain soulagement et aussi d'une certaine autosuggestion sur la situation politique en Algérie. En 1961, Mademoiselle Fatima Dekhinet, première adjointe d'une ville de l'arrondissement, décorée de la croix de la Valeur militaire, passe à Angers, Cholet, Saumur. Mais la visite de la délégation officielle de Barika est plusieurs fois repoussée : automne 1960, printemps 1961, mai 1961. Elle se révèle finalement "impossible en raison de la conjoncture ${ }^{35}$ ", ce qui n'empêche pas la mise en œuvre du jumelage.

\section{Des actions humanitaires et solidaires}

\section{L'encadrement et le financement par les autorités}

Les instructions officielles et la propagande insistent beaucoup sur la dimension humaine des jumelages, les départements orientent nettement les actions vers l'humanitaire. Ceux-ci sont présentés comme devant favoriser un développement social, la promotion des populations. Pour tendre à cela, il faut mobiliser les énergies et les moyens. Si le dynamisme des réseaux associatifs de l'Ouest s'exprime dans le cadre des jumelages, l'impulsion des conseils généraux est déterminante, notamment sur le plan financier. Celui de la Sarthe vote dès l'automne 1959 une subvention d'un million d'anciens francs qui doit servir "à des travaux de coût modique dont l'utilité sera immédiatement appréciée des habitants ". Effectivement, une fontaine est construite, un accès empierré est ouvert, de la nourriture est distribuée... En avril 1960, une nouvelle subvention de 54000 NF est destinée à construire un petit dispensaire et à creuser cinq autres points d'eau. Le département de la Mayenne vote une subvention de $600000 \mathrm{AF}$ et Laval accorde $300000 \mathrm{AF}$ pour Périgotville. Une association MayenneKerrata est créée pour gérer ces fonds et les collectes de vivres, médicaments et vêtements organisées par les antennes locales des acteurs humanitaires. Du Mans partent 16 caisses de vêtements collectés par la Croix-Rouge, le Secours Catholique et les compagnons d'Emmaüs. Mais de grands projets sont toujours repoussés comme la lutte contre l'érosion des terres sur place ${ }^{36}$. Au printemps 1961, face à la sécheresse qui touche l'est de l'Algérie et à la faim qui commence à toucher les populations, le préfet de Constantine demande du blé, de la semoule et des vêtements, " une aide dans la bataille que nous livrons, ici, au nom de la France, contre

34. Arch. dép. de Maine-et-Loire, 303 W 376, compte rendu du voyage d'étude effectué du 2 au 7 mai par la délégation du conseil général; " Envoyés par le conseil général dans l'arrondissement filleul de Barika, MM. De Boischevalier, Loire et Ossant sont revenus convaincus de la confiance des musulmans en la France ", La Nouvelle République, 11 mai 1960 .

35. Arch. dép. de Maine-et-Loire, 303 W 376, lettre du sous-préfet de Barika au préfet de Maine-et-Loire, 31 mai 1961.

36. Foucault, Rémy, " Mayenne-Kerrata (1959-1962)... ", art. cit., p. 11 et 15. 
la misère des populations ${ }^{37}$ ". Ainsi, se manifeste, notamment de la part de certains élus et d'acteurs associatifs sensibilisés à la question du tiersmonde, un intérêt pour des actions de développement à moyen ou long terme. Mais ce n'est pas l'aspect le plus répandu.

Dans le contexte de la guerre, la dimension militaire des jumelages est encouragée par plusieurs acteurs. L'association Rhin et Danube qui réunit les anciens de la $1^{\text {re }}$ Armée Française consacre un dossier de son journal à l'opération (septembre 1960). De même, la Fondation Maréchal de Lattre fait paraître un numéro spécial de son bulletin de liaison afin de développer dans le cadre des jumelages le soutien aux soldats français. La campagne 1960 de la Fondation s'appuie sur le slogan : "Votre département doit aussi aider les soldats qui veillent sur l'arrondissement algérien ou saharien jumelé ". Elle plaide pour qu'une partie des aides accompagnant les jumelages finance des actions encadrées par l'armée, les écoles du bled notamment ${ }^{38}$. Grâce à la Fondation, l'Amicale des anciens d'Afrique du Nord de Cholet envoie des vêtements et un colis d'antibiotiques. Les officiers de réserve s'engagent fortement dans l'opération. Certains visitent les arrondissements algériens et sont des aiguillons qui poussent les départements à agir. Ainsi, trois d'entre eux sont à Barika à la fin de 1960 et rédigent un rapport très critique sur les promesses non tenues faites par la délégation du Maine-et-Loire. Accusé de faire moins d'efforts que d'autres, le président du conseil général est contraint de démentir des "Propos fantaisistes". Le département n'a-t-il pas envoyé une machine à coudre qui permettra d'apprendre à coudre à des filles ${ }^{39}$ ?

Les conseils généraux sont aussi sous la pression des préfets qui font en sorte que leur département ne soit pas en reste. En avril 1960, le préfet demande au maire d'Angers s'il accepte de jumeler sa ville avec celle de Barika, " car il a été décidé, dans un but d'harmonisation, que les parrainages entre communes devaient s'effectuer dans le cadre des jumelages départements-arrondissements algériens ${ }^{40} "$. Le maire est d'accord, mais veut savoir ce que le Maine-et-Loire fait concrètement..., ce qui ne doit donc pas aller de soi. La décision du conseil municipal est positive le 26 septembre 1960, seul un conseiller s'abstient car il est " contre le jumelage avec la ville d'un pays dont on ne connaît pas le statut futur ${ }^{41}$ ". Le préfet de la Sarthe organise quant à lui le jumelage des principales communes du département avec des communes de l'arrondissement de Kerrata. Il envisage même de dé-jumeler Château-du-Loir puisque Thiersville fait partie de l'arrondissement d'Oran qui est jumelé avec la Seine-et-Oise. Mais les deux

37. Arch. dép. de Maine-et-Loire, 303 W 376, lettre du préfet de Constantine, 30 mai 1961.

38. Arch. dép. de la Sarthe, 1359 W 137, Fondation Maréchal de Lattre, Bulletin $\mathrm{n}^{\circ} 42$, 21 juin 1960.

39. Arch. dép. de Maine-et-Loire, 303 W 376, lettre au préfet, 7 janvier 1961 et réponse du 25 janvier.

40. Arch. nat. d'Outre-mer, $1 \mathrm{~K} 1288$, liste des parrainages de communes, 2 mai 1959.

41. Arch. mun. d'Angers, délibération du conseil municipal, 26 septembre 1960; Arch. dép. de Maine-et-Loire, 303 W 376, lettre du SGAA, 5 novembre 1960. 
villes refusent. Sur les 13 communes sollicitées par le préfet, seulement quatre ont répondu positivement; le conseil municipal de la Ferté-Bernard " a prié le Maire de n'engager aucunement la commune dans cette affaire ${ }^{42}$ ".

\section{Des mobilisations humanistes surtout en faveur de l'enfance et de la jeunesse}

La situation de l'enfance et de la jeunesse est une composante importante de la question sociale qui est exacerbée par la guerre d'indépendance algérienne. Les autorités françaises mobilisent la population de la métropole sur ce thème, faisant de l'enfance un vecteur de l'aide aux populations déshéritées d'Algérie, dans une configuration traditionnelle de rapports coloniaux inégalitaires. L'enfance et la jeunesse constituent une cause - en tant que victimes innocentes - en même temps qu'un enjeu fort de la guerre psychologique en Algérie car il ne saurait être question de les laisser entre les mains des indépendantistes.

Le ministre de l'Éducation nationale incite à des jumelages d'écoles qui sont également encouragés par le SGAA après une exposition présentée à Paris, intitulée "La jeunesse d'Algérie vous parle ". Le journal Tintin et la radio musulmane d'Alger lancent une opération d'échanges épistolaires. En Loire-Atlantique, les enfants des écoles envoient des lettres à leurs homologues de Paul-Cazelles ${ }^{43}$. En Vendée, des écoles participent à l'envoi de livres pour des "écoles militaires " de Khenchela qui se font sous la tente par des appelés ${ }^{44}$. En 1960, la presse angevine publie des articles sur Barika, en insistant sur la pauvreté et le manque de moyens : sur 21550 enfants que compte l'arrondissement, 1630 seulement vont en classe, 11 communes sur 14 n'ont pas d'école. L'année suivante, la jeune chambre économique d'Angers lance une " aide culturelle aux enfants d'Algérie " afin de combler le " déséquilibre certain qui existe entre l'enfant français et l'enfant algérien sur le plan de l'éducation intellectuelle ". Une collecte de livres de classe associe l'inspection académique et les enseignants ${ }^{45}$.

Le ministère d'État chargé des Affaires algériennes soutient l'action de l'association "Les étrennes du Bled ", qui vise à distribuer aux enfants défa-

42. Arch. dép. de la Sarthe, 1359 W 137, situation des jumelages entre communes, 8 février 1961; La Nouvelle République, 20 janvier 1961. Finalement, la commune des Amouchas est jumelée avec les communes des cantons de Malicorne et de Sablé. Son maire est reçu dans la Sarthe en janvier 1961.

43. Arch. du ministère des Affaires étrangères, circulaire du ministère de l'Éducation nationale, 7 décembre 1959; SGAA, $1^{\mathrm{er}}$ mars 1960; Arch. dép. de Loire-Atlantique, $270 \mathrm{~W}$ 335, lettre du préfet, 25 mars 1960.

44. Arch. dép. de la Vendée, 1494 W 659, sous-préfet de Khenchela au préfet de Vendée, 29 octobre 1960.

45. "Quelques aspects de Barika ", Le Courrier de l'Ouest, 12-13 février 1960, et surtout : "Angevins, voici le Barika, votre filleul ", " Jumelé avec le Maine-et-Loire, l'arrondissement algérien de Barika se présente à nos lecteurs ", 16 février 1960 : "Pour les jeunes Algériens de Barika. La Jeune Chambre économique lance l'opération "ramassage de livres" ", Le Courrier de l'Ouest, 4 mars 1961. 
vorisés d'Algérie des jouets et jeux collectés par des enfants de la métropole, notamment par l'intermédiaire des clubs de sport. Des cartes postales sont ainsi vendues dans les associations sportives de Loire-Atlantique et le conseil général vote une subvention de $100000 \mathrm{AF}^{46}$. La Croix-Rouge des Deux-Sèvres envoie en décembre 1959 pour 150000 AF de couverture et en 19611500 paires de chaussures, un organisme médical 150000 francs de boîtes de lait et autres produits de nécessité. Tous ces envois sont à destination des enfants des camps de regroupement forcé de population à Collo $^{47}$. De même, le sous-préfet de Paul-Cazelles reçoit au printemps 1960 63 colis de vêtements envoyés par la Croix-Rouge de Loire-Atlantique ${ }^{48}$. Des layettes sont expédiées par le centre hospitalier du Mans; une vente de sucre de la Cimade rapporte $350 \mathrm{NF}$; le Secours Catholique organise une opération " Noël quand même " qui récolte 500 NF destinés " à gâter un peu les enfants de cette région adoptée ${ }^{49} "$.

Le dynamisme de certains jumelages doit beaucoup à des personnalités politiques fortes qui s'y engagent pleinement, notamment pour des raisons politiques : le Premier ministre Michel Debré, implanté à Amboise, suit de près le jumelage de l'Indre-et-Loire, tout comme Jacques Chaban-Delmas en Gironde ou Robert Buron en Mayenne ${ }^{50}$. À un échelon plus local, en Sarthe, la comtesse Armand, qui a été résistante et membre du bureau de l'OCM (Organisation civile et militaire), conseiller général de Malicorne, est " l'âme du jumelage " avec Kerrata. Elle a perdu son fils Étienne, appelé en Algérie, dans un accident en février 1959, et elle poursuit l'action qu'il avait lancée en faveur des enfants déshérités. En mars 1960, avec l'association des veuves et orphelins de guerre qu'elle préside, elle organise des envois de lait, médicaments et friandises pour les enfants. En juin 1960, elle est en Algérie, accompagnée de Saïd Belhahhaf, un manceau qui a été liquidateur de l'OCM en Sarthe et qui participe également activement aux parrainages de villes et au jumelage ${ }^{51}$.

Des individualités plus discrètes ouvrent également pour les enfants. Après 25 mois en service en Algérie, Édouard Le Floc'h, un appelé sarthois, instituteur à Château-du-Loir, revient en métropole en ramenant avec lui Mohamed, un orphelin de 13 ans de la région de Biskra, recueilli et gardé

46. Arch. dép. de Loire-Atlantique, 270 W 335, lettre du ministre d'État du 17 décembre 1960, réponse du préfet du 25 janvier 1961, cartes postales " Aller-et-retour ".

47. Arch. dép. des Deux-Sèvres, 1206 W 1, procès-verbal du 12 janvier 1960; lettre du conseil départemental de la Croix-Rouge des Deux-Sèvres, 12 avril 1961.

48. Arch. dép. de Loire-Atlantique, 270 W 335, lettre du sous-préfet de Paul-Cazelles au président du conseil général, 5 mai 1960.

49. Arch. dép. de la Sarthe, 1359 W 137, correspondance des associations et articles de journaux, 1960.

50. BouRdin, Gérard, "Les ambiguïtés d'un rapprochement... ", dans BRANCHE, Raphaëlle, ThÉNAUlT, Sylvie (dir.), La France en guerre..., op. cit., p. 82-83.

51. " $\mathrm{M}^{\mathrm{me}}$ la comtesse Armand et l'Association des veuves de guerre viennent en aide aux écoliers des Amouchas ", Le Maine Libre, 26 mars 1960; "Le voyage en Algérie de Mme la comtesse Armand ", Ouest-France, 23 juin 1960; "La visite en Algérie de Mme la Comtesse Armand ", Le Maine Libre, 24 juin 1960. 
pendant six mois par sa compagnie ${ }^{52}$. L'enfant a appris le français, suivi le rythme de la vie militaire et a été blessé lors d'un harcèlement du poste par l'ALN. Bien qu'il ait encore de la famille collatérale, le cadi (juge) a accepté de confier l'enfant à l'instituteur, pas encore en âge de l'adopter, contre la promesse d'assurer sa subsistance et son éducation. D'après la presse, Mohamed s'est intégré immédiatement dans sa nouvelle famille (la mère et le beau-père d'Édouard) et il est prévu que l'enfant aille à l'école du village où « il sera reçu comme un enfant du pays ». L'article est aussi l'occasion de souligner que dans les régions les plus déshéritées d'Algérie, la présence de l'armée française a contribué à l'amélioration du sort misérable des enfants musulmans. Édouard émet l'idée que des enfants musulmans puissent être accueillis en Sarthe pour l'été ${ }^{53}$.

Le séjour en métropole d'enfants et de jeunes d'Algérie est très tôt posé comme un des objectifs à réaliser, une action déterminante pour l'avenir. Le Bled, journal d'information militaire à destination des soldats, signale qu'en juillet 1957 « 500 enfants d'Algérie, européens et musulmans, passent leurs vacances en France, dans des familles qui les ont invités ", surtout des familles nombreuses des départements de l'Est et du Nord ${ }^{54}$. En août 1960, l'office départemental des anciens combattants de Sarthe organise le séjour de 20 pupilles de la nation de Constantine et de Kerrata : ils sont placés dans des familles et dans une colonie de vacances, grâce au financement du ministère des Anciens Combattants et à la générosité d'associations. À la rentrée 1961, 52 enfants français-musulmans d'Algérie sont accueillis en formation dans 17 maisons familiales rurales, dont à Brehoulou (Finistère), aux Trois-Croix (Ille-et-Vilaine) et à Pétré (Vendée) ${ }^{55}$.

\section{L'action phare des jumelages : les colonies de vacances}

\section{Économie générale des colonies de vacances}

Après plusieurs expériences durant l'été 1959 (notamment en DeuxSèvres et en Indre-et-Loire), le SGAA invite les préfets à organiser des colonies de vacances de jumelage à l'été 1960. L'Office Algérien des Colonies de Vacances (OACV) est chargé du plan de transport jusqu'à Marseille, financé par le Gouvernement général, à hauteur de 100 enfants par arron-

52. D'autres exemples de ces enfants recueillis par l'armée dans DENÉcHÈRE, Yves, « Les "enfants de Madame Massu" : œuvre sociale, politique et citoyenneté pendant et après la guerre d'Algérie (1957-1977) " [article à paraître dans la Revue d'Histoire Moderne et Contemporaine]. Un petit kabyle est recueilli pendant 18 mois puis pris en charge par une association. Un autre, également récupéré par l'armée, est ensuite adopté par le général Massu et son épouse.

53. "Édouard Le Floc'h de Marçon a ramené des Aurès un orphelin musulman ", OuestFrance, 11 avril 1960.

54. "Vacances en France ", Le Bled, nº 68, 27 juillet 1957.

55. Arch. dép. de la Sarthe, 1359 W 137, "Exposé des réalisations Sarthe-Kerrata ", automne 1960; Arch. dép. de la Vendée, 1494 W 659, lettre du ministre de l'Intérieur aux préfets concernés, 27 septembre 1961. 
dissement jumelé ${ }^{56}$. Ces colonies sont considérées comme " un des résultats les plus tangibles des jumelages ". En 1960, plus des deux tiers des départements jumelés en organisent : 3266 enfants, adolescents et accompagnateurs séjournent ainsi en France. L'encadrement de l'opération est très strict : pour l'Ouest, le conseiller technique des affaires musulmanes de la III $^{\mathrm{e}}$ région (Rennes) demande à être tenu au courant de l'organisation de chaque colonie et à connaître " tout incident éventuel d'une certaine importance ${ }^{57}$ ".

La dimension de propagande des colonies de vacances est évidente. Le journal social bimensuel Messages d'Algérie écrit aux enfants partant en colonie pour leur demander d'envoyer un récit et un dessin de leur séjour pour publication. En août 1960, le ministère de l'Intérieur demande une documentation (photographies, dessins, etc.) sur les colonies accueillies dans chaque département, " pour être utilisée à illustrer l'effort fait en faveur de l'enfance algérienne ". Le préfet de Loire-Atlantique envoie aussi des vanneries, tissages, poteries et autres colliers confectionnés par les enfants ${ }^{58}$.

Pour l'année 1961, les statistiques de l'OACV font état de 3589 enfants envoyés en colonies de vacances, avec 311 moniteurs dans le cadre de 87 jumelages d'arrondissements et de 63 départements (et de quelques villes) ${ }^{59}$. Les préfets et conseils généraux sont donc à l'œuvre puisque l'organisation d'une colonie de vacances est le signe d'un jumelage qui fonctionne. En janvier 1960, un élu des Deux-Sèvres de retour de Collo, faisant le constat de l'état misérable des enfants, propose une action d'une grande ampleur : la venue en Deux-Sèvres pendant trois semaines de « 300 garçons et d'environ 80 à 100 filles, âgés de 9 ans à 14 ans, sous-alimentés, las de crainte et d'inquiétude ". Il est même question que " chacun des 31 cantons des Deux-Sèvres [reçoive] l'été prochain [1960] en colonie 20 enfants de l'arrondissement jumelé ". L'idée vient de l'épouse du sous-préfet de Collo qui s'occupe de toutes les actions sociales. Le projet, évalué à $4200000 \mathrm{AF}$, donne lieu à un débat au cours duquel certains rappellent leur opposition de principe au jumelage - renforcée par le choix gaullien de la voie de l'autodétermination qui vise à l'expression libre des habitants d'Algérie - et propose que cette somme soit plutôt consacrée à venir en aide aux populations regroupées de force dans l'arrondissement de Collo. Ainsi la somme profiterait directement et entièrement aux populations concernées, alors que le seul transport des enfants venant en colonie engloutirait la moitié de la somme. L'unanimité est cependant acquise. Mais la règle de l'OACV

56. Arch. nat., F1 cII 546, circulaire du SGAA aux préfets, 24 décembre 1959.

57. Arch. dép. de Loire-Atlantique, 270 W 335, lettre du préfet d'Ille-et-Vilaine au préfet de Loire-Atlantique, 17 août 1960.

58. Arch. dép. de Maine-et-Loire, 303 W 376, lettre du 17 juillet 1961; tract des Messages d'Algérie distribué aux enfants des colonies; Arch. dép. de Loire-Atlantique, 270 W 335, lettre du préfet de Loire-Atlantique, 24 septembre 1960.

59. Arch. dép. de Maine-et-Loire, 303 W 376, statistiques de l’OACV sur les colonies de jumelages pour l'année 1961. 
est de prendre en charge le transport jusqu'à Marseille pour 100 enfants maximum. Après avoir été révisé à 150 en juin, le nombre d'enfants est arrêté à 90, plus 10 moniteurs algériens. Les enfants sont tous musulmans et scolarisés : 61 garçons sont hébergés dans l'école normale de Parthenay et 29 filles à la maison familiale des Aubiers et à l'école ménagère de SaintePézenne. Au final le coût s'élève à 25300 NF ${ }^{60}$.

En août 1960, 90 enfants (garçons et filles) de l'arrondissement de Kerrata sont en colonie de vacances en Mayenne (38 à Laval, 37 à Mayenne, 15 à Château-Gontier). Les autorités sont très présentes, notamment lors de l'accueil solennel par le préfet. La couverture de la presse locale est forte : 10 articles et 13 photos rien que dans Ouest-France. Selon le quotidien, l'accueil de la population est bon : les enfants sont mélangés avec les enfants du pays dans les garderies urbaines, participent à des excursions le dimanche, dont une à Saint-Malo offerte par la Croix-Rouge. Le feu de camp d'adieu à Mayenne a lieu sur le champ de foire. L'année suivante, 93 enfants de Kerrata sont accueillis, tous à Laval, l'enthousiasme est moindre comme en témoignent les rares articles dans la presse locale ${ }^{61}$. Il faut dire que le contexte a bien changé : le référendum sur l'autodétermination en Algérie a eu lieu le 8 janvier 1961, le putsch des généraux en avril, les troupes françaises commencent leur retrait...

La Loire-Atlantique accueille en 1960 une centaine d'enfants de PaulCazelles qui arrivent par avion à l'aéroport de Château-Bougon où les attendent de nombreuses autorités. Le département, la ville de Nantes et la chambre de commerce financent l'opération sur place. Les enfants sont répartis par petits groupes dans une demi-douzaine de colonies de vacances sur la côte (Saint-Brévin, Préfailles, Le Croisic, Tharon...) organisées par l'Association catholique nantaise des colonies de vacances, qui fixe le " prix de revient d'une journée d'enfant algérien " à 9 NF. Un plus grand groupe (45) intègre la colonie internationale de la Fédération des Amicales Laïques à Bonne Anse (Saint-Nazaire) où se trouvent aussi 20 Yougoslaves ${ }^{62}$. Un autre contingent est accueilli à la colonie protestante de la Brise de Mer à Saint-Michel-Chef-Chef. Les organismes laïque, catholique et protestant sont donc tous mobilisés. Une autre colonie de 50 enfants, en marge du jumelage, est organisée à Vertou par deux anciens appelés nantais et angevin ${ }^{63}$. Pour 1961, un budget de près de $80000 \mathrm{NF}$ est prévu par le département. Début août 1961, ce sont 124 enfants de 9 à 14 ans de Paul-Cazelles qui repartent en avion de Nantes : 32 fillettes de la

60. Arch. dép. des Deux-Sèvres, 1206 W 1, procès-verbal de la séance du CG du 12 janvier 1960 ; rapports du préfet du 16 juin et 21 septembre 1960 ; rapports des renseignements généraux des $1^{\mathrm{er}}$ et 15 juillet 1960 .

61. Foucault, Rémy, " Mayenne-Kerrata (1959-1962)... ", art. cit., p. 12-14.

62. " De jeunes colons algériens sur nos plages ", L'Éclair de l'Ouest, 5 août 1960; "Cent petits Algériens accueillis à Château-Bougon ", La Résistance de l'Ouest, 5 août 1960.

63. Sur cette colonie et d'autres accueils d'enfants et de jeunes, voir DENÉCHÈRE, Yves, "Enfants et jeunes musulmans de l'Algérie en guerre dans le Maine-et-Loire (1959-1961)", Archives d'Anjou, no 19, 2017, p. 152-165. 
ville d'Alger qui étaient en colonie à Plouescat (Finistère) en juillet et 92 garçons de Paul-Cazelles qui étaient dans des colonies de Loire-Atlantique ${ }^{64}$.

En Vendée, en 1961, deux groupes d'enfants sont accueillis dans le cadre du jumelage : d'une part, " 9 enfants sélectionnés parmi les meilleurs élèves des établissements scolaires " qui viennent passer 10 jours de découverte aux Sables-d'Olonne et aux environs; d'autre part, 44 enfants qui seront en colonie pendant un mois ${ }^{65}$. Dans les Deux-Sèvres, la couverture de presse est intense, notamment en raison de la présence importante des personnalités politiques et administratives qui viennent visiter les colonies. Les renseignements généraux suivent le séjour de près. Un ouvrier installé à Nantes, originaire de Collo, vient voir sa nièce venue en colonie... après enquête et avec une autorisation en bonne et due forme ${ }^{66}$.

Parfois, la couverture médiatique est plus polémique, comme c'est le cas pour une colonie hors jumelage de l'OACV à Lorient en 1959. D'après La Liberté du Morbihan, les 64 enfants de Tlemcen sont désœuvrés, mal encadrés et mal pris en charge, l'opinion publique s'en émeut. L'abbé Laudrin s'en mêle - car " mes collègues de l'Assemblée Nationale ne me pardonneraient jamais d'avoir abandonné ces gosses ", affirme-t-il. Il est en effet le dernier ecclésiastique élu député. En tant que directeur de la colonie des "Casques blancs " l'abbé " incorpore " ces enfants aux siens... Cette présentation des choses est plus que tempérée par l'inspecteur d'académie qui signale que tous les services de l'État ont été mobilisés pour accueillir au mieux ces enfants qui n'étaient en rien abandonnés, et à demi-mot de laisser penser à une sorte de récupération politique ${ }^{67}$.

Le conseil général de Maine-et-Loire prépare une colonie de vacances pour 1961. Consultée, la Fédération départementale des associations Famille rurale se déclare " très favorable à la participation de l'accueil de jeunes algériens dans les familles de notre Anjou rural ", et d'insister sur la sélection des familles accueillantes pour " recevoir et traiter dignement ces enfants". La colonie est prévue pour 50 enfants, 25 garçons et 25 filles, de 8 à 14 ans. Mais le sous-préfet de Barika estime dès le mois de mars qu'il aura du mal à atteindre ce nombre : «Ma prospection se révèle d'ores et déjà assez difficile plus particulièrement en ce qui concerne les filles ". La liste des enfants tarde également à arriver en raison de " la réticence rencontrée chez de nombreux parents ${ }^{68}$ ". Malgré toute une politique visant à faire changer les mentalités, dont l'ordonnance du 4 février 1959 sur « l'évolution de la femme

64. Arch. dép. de Loire-Atlantique, extraits d'articles de presse des 5 et 7 août 1961, titres inconnus.

65. Arch. dép. de la Vendée, 1494 W 659, note à l'attention du préfet, s. d.

66. Arch. dép. des Deux-Sèvres, 1206 W 1, rapports du préfet du 16 juin et 21 septembre 1960 ; rapports des renseignements généraux des $1^{\text {er }}$ et 15 juillet 1960 . Une vingtaine d'articles au moins dans La Nouvelle République, Ouest-France et Le Courrier de l'Ouest.

67. Arch. nat., 19770178/26, La Liberté du Morbihan, 8 août 1959; rapport de l'inspecteur d'Académie du 31 août 1959.

68. Arch. dép. de Maine-et-Loire, 303 W 376, « Organisation de colonie de vacances pour les jeunes de Barika ", s.d.; correspondance du sous-préfet de Barika, mars-juin 1961. 
musulmane en Algérie " et l'organisation de séjours de jeunes filles en France sous le nom de "Caravanes musulmanes ", les parents rechignent à laisser partir leurs filles, surtout après l'âge de 9-10 ans ${ }^{69}$.

Finalement, du 5 juillet au 4 août 1961, 46 colons de 8 à 14 ans (33 garçons et 13 filles), présentés comme les meilleurs élèves des écoles primaires de Barika, sont accueillis à la maison familiale de Beaupréau. Une jeune monitrice qui suit des cours d'infirmière en France parle arabe et sert d'interprète car les enfants parlent mal français. Les encadrants se souviennent de la difficulté à faire respecter l'hygiène, notamment parce que les enfants n'ont pas l'habitude d'utiliser des commodités, ni d'aller à la piscine. Mais il n'y a pas de situation délicate à régler ou de tension particulière ${ }^{70}$. Des familles de Beaupréau et de Cholet accueillent un enfant pour une journée ${ }^{71}$. Le conseil général est très présent, mobilise des moyens, souhaite "faire œuvre utile ", veille à ce que tout se passe bien, afin "que les enfants gardent une bonne image des Français ". Les enfants sont très suivis par les services sociaux, des médecins, des assistantes sociales. Un moniteur se souvient qu'il y avait " des barrières partout ", par exemple il était interdit d'aller au parc voisin. Tout cela est très cadré, avec beaucoup de sorties très organisées, mais finalement peu de contacts avec la population, surtout de la communication lors des visites. Les élus souhaitent le passage des enfants dans leurs communes pour que la presse locale en parle.

\section{Les effets ici et là-bas}

Après le départ des enfants de Barika, au cours d'un rapide bilan, tous les acteurs (officiels, encadrants, associations) manifestent un réel soulagement et leur satisfaction en constatant que l'" opération ", malgré " des conditions difficiles ", s'était à peu près bien déroulée ${ }^{72}$. Le sous-préfet de Barika rend compte du retour des enfants en évoquant la "vive gratitude " des parents et élus vis-à-vis de "l'affectueuse sollicitude de l'encadrement ". De même, le maire de Zoui, l'une des communes les plus déshéritées de l'arrondissement de Khenchela, remercie le conseil général de Vendée pour l'accueil d'un des orphelins musulmans de sa commune. Le maire de Khanga-Sidi-Nadji, " très reconnaissant de tout ce que le département de Vendée a fait pour notre commune " propose au préfet de lui envoyer 10 quintaux de dattes muscades; celui-ci répond qu'elles seront distribuées

69. ZERDoumi, Nefissa, Enfants d'hier. L'éducation de l'enfant en milieu traditionnel algérien, Paris, François Maspero, 1970, "L'éducation des petites filles », p. 185-192; SAMBron, Diane, "L'évolution du statut juridique de la femme musulmane à l'époque coloniale ", Histoire de la justice, 1/2005 (no 16), p. 123-142.

70. Témoignages de Pierre Mainguy, directeur de la colonie - Angers, 18 février 2016, et de Paul Bridonneau, moniteur de la colonie - Angers, 16 mars 2016.

71. « Les colons de Barika actuellement à la maison familiale de Beaupréau ont passé la journée d'hier dans les familles d'accueil ", Le Courrier de l'Ouest, 26 juillet 1961.

72. Témoignage de Pierre Mainguy. 
aux enfants des écoles vendéennes ${ }^{73}$. Dans une même volonté de réciprocité, le maire de Périgotville avait bien proposé d'accueillir des enfants lavallois dans sa ville, mais ce fut impossible car leur sécurité n'aurait pas été garantie ${ }^{74}$.

Une directrice de colonie de Loire-Atlantique souligne les très bonnes relations avec les enfants locaux : "Nos enfants ont été très heureuses d'avoir des camarades d'outre-mer. Certains parents leur ont même offert des petits souvenirs et des friandises. Voilà une expérience que nous serons très heureux de renouveler. " Mais le préfet signale que si dans certaines colonies les enfants étaient bien mélangés avec les autres, à Bonne Anse ils étaient seulement entre eux, regroupés dans une annexe de la colonie, " ce qu'il eût fallu éviter. Par ailleurs, cette colonie accueillant nombre de Yougoslaves et d'Allemands, il est à craindre que les petits Algériens ne s'estiment traités en étrangers ". Les enfants ont chanté un chœur en arabe, ce qui est considéré comme " un incident " à signaler. Mais sur l'ensemble des centres, "l'impression est excellente : les enfants paraissent complétement acclimatés et intégrés ${ }^{75}$ ".

Le préfet des Deux-Sèvres dresse un long bilan de cette " action sociale et psychologique " qu'il estime très positive sur tous les plans. Il n'y a eu aucun problème sanitaire à signaler, si ce n'est quelques rhumes et quelques poux. Il insiste surtout sur l'effet psychologique sur les enfants : ils ont été contents " de vivre sans avoir peur ", même si les filles ont été plus passives que les garçons, tout en réalisant des objets présentant " une étonnante facture artistique ". Du côté des populations locales, le préfet signale de la sollicitude, de la sympathie, de l'intérêt, de l'amitié, surtout aux Aubiers, " dans un milieu essentiellement rural très attaché aux traditions familiales du catholicisme ". Un cahier photographique du séjour a été réalisé. Les renseignements généraux du département signalent notamment que " la population a réservé à ces enfants le meilleur accueil et en règle générale l'opinion publique se montre favorable à leur séjour dans notre département. Ils ont participé à la retraite aux flambeaux du 14 juillet ". Aux Aubiers en particulier, des contacts étroits ont eu lieu entre enfants et familles du pays. Beaucoup de celles-ci pensent que ce voyage a été profitable aux enfants car il leur a permis " de voir et de connaître un coin de cette France dont ils entendent parler ". Des contacts ont vraiment lieu entre colons de Collo et enfants des DeuxSèvres, notamment lors d'un " rallye de la Paix " à Chatillon-sur-Sèvre, regroupant 500 enfants de la commune, de Paris et d'Algérie. Organisés

73. Arch. dép. de Maine-et-Loire, 303 W 376, lettre du sous-préfet de Barika au préfet de Maine-et-Loire, 10 août 1961; Arch. dép. de la Vendée, 1494 W 659, lettre du maire de Zoui, 7 octobre 1961; lettre du maire de Khanga-Sidi-Nadji, 22 décembre 1961 et réponse du préfet, 28 décembre.

74. Foucault, Rémy, " Mayenne-Kerrata (1959-1962)... ", art. cit., p. 11.

75. Arch. dép. de Loire-Atlantique, 270 W 335, lettre du préfet d'Ille-et-Vilaine au préfet de Loire-Atlantique, 17 août 1960; réponse du 24 septembre 1960; rapport de visite, septembre 1960 . 
par le Mouvement de l'Enfance, le pique-nique et le « jeu international de la paix " ont été précédés par une messe ${ }^{76}$.

Un bilan dressé par l'OACV sur la campagne 1960 s'attarde sur l'accueil des colonies par la population en Bretagne. Un directeur de colonie rapporte :

"Le Breton passe pour être renfermé et hostile à qui n'est pas de sa religion; aussi l'accueil réservé à nos enfants m'a-t-il surpris agréablement... À aucun moment nous n'avons dû déplorer le moindre signe de froideur. Bien au contraire, les témoignages de sympathie abondent et il est évident que nos gamins n'avaient pas l'habitude d'évoluer dans un pareil climat d'amitié. À Doëlan-sur-Mer un marin pêcheur explique aux enfants les subtilités de la pêche aux crabes. Mais le plus beau souvenir de la colonie restera pour tous sans doute la kermesse organisée par la colonie de Puteaux où étaient conviés les habitants de Plœmeur. Jamais nos petits n'avaient connu une pareille liesse : bal, stands, marchand de frites et ce monôme final réunissant adultes et enfants auquel tous ont participé. Cette atmosphère de 14 juillet reconstitué en pleine Bretagne les a conquis d'emblée. Populations et ambiance générale de la colonie ont donc contribué à faire de la colonie un merveilleux souvenir ${ }^{77}$."

De l'avis général, les colonies " ont laissé une impression profonde dans l'esprit des jeunes musulmans ${ }^{78}$ ".

Il est intéressant de constater que l'impact du contexte politique de la fin de la guerre sur l'organisation des colonies de vacances est très différent d'un département à un autre. Alors que le Maine-et-Loire organise sa première colonie en 1961, cette année-là le conseil général des Deux-Sèvres, « a jugé opportun, en raison des circonstances actuelles, d'ajourner jusqu'à sa prochaine session, toute décision concernant sa participation au jumelage en 1961 ", mais le préfet émet néanmoins l'idée d'organiser la même colonie qu'en 1960 et prend des initiatives dans ce sens. Finalement, le projet est abandonné au profit d'une autre action. Une grande sécheresse touchant la région de Collo, le conseil général décide que la somme de 30000 francs prévue pour accueillir 80 enfants en colonie sera versée directement à l'arrondissement pour venir en aide aux victimes les plus sévères de la sécheresse ${ }^{79}$. Ce qui témoigne encore une fois de la volonté du département de décliner son jumelage comme il l'entend.

Après la réussite de la colonie de 1961, le Maine-et-Loire en prévoit une pour 1962, mais l'organisation traîne alors que les accords d'Évian sont

76. Arch. dép. des Deux-Sèvres, 1206 W 1, rapports du préfet au ministère de l'Intérieur et au préfet de Constantine, 4 août et du 21 septembre 1960 ; rapports des renseignements généraux des $1^{\mathrm{er}}$ et 15 juillet 1960; La Nouvelle République et Le Courrier de l'Ouest, 17 juillet 1960 .

77. Arch. nat., 19770178/26, Compte rendu d'activités pour 1960, OACV, extrait d'une lettre d'un directeur.

78. Arch. dép. de Maine-et-Loire, 303 W 376, le ministre d'État chargé des Affaires algériennes aux préfets, 10 janvier 1961 .

79. Arch. dép. des Deux-Sèvres, 1206 W 2, rapport du préfet, 31 janvier 1961 ; correspondance entre les préfets des Deux-Sèvres et de Constantine; "Pas de vacances dans les Deux-Sèvres pour les enfants de Collo, cette année ", La Nouvelle République, 14 juin 1961. 
signés en mars Au mois de juin, le préfet interroge le conseil général, sans cacher " les grandes difficultés " liées au calendrier politique : " La colonie est prévue au mois d'août, après le vote sur l'autodétermination [référendum d'autodétermination du $1^{\mathrm{er}}$ juillet]. Il est vraisemblable qu'à cette date, les autorités responsables dans le département des Aurès et l'arrondissement de Barika ne seront plus les mêmes "; il propose de reporter la colonie à une année ultérieure. Pense-t-on alors que le jumelage pourra se poursuivre? En tout cas l'abandon du projet est très tardif, bien après le cessez-le-feu du 19 mars 1962, il n'est donc pas lié aux opérations militaires, ni à la guerre. Finalement, c'est la concomitance de l'accueil des enfants et l'arrivée des Français d'Algérie qui inquiète : " La présence des petits algériens pourraient provoquer avec des rapatriés des difficultés qu'il est préférable d'éviter ${ }^{80}$." Le projet est donc abandonné sine die. En Vendée, jusqu'en juin 1962, le préfet prend toutes dispositions pour organiser une colonie de vacances dans les mêmes conditions qu'en 1961. Mais le sous-préfet de Khenchela lui indique le 29 juin que c'est impossible : il doit quitter, avec ses principaux collaborateurs, son poste dans les jours qui suivent, " dès lors l'organisation d'une colonie est parmi les questions qui ne pourront être traitées que par nos remplaçants, dans le cadre de la nouvelle souveraineté ${ }^{81}$ ". Quelques jours plus tard, le 3 juillet, l'indépendance de l'Algérie est proclamée.

Les autorités françaises appellent les communes et les départements jumelés à participer à l'effort d'accueil des rapatriés. C'est clairement une réorientation de l'action. Le ministre des Affaires algériennes et le secrétaire d'État aux Rapatriés indiquent qu'en ce qui concerne les colonies de 1962 " les enfants musulmans que leurs familles souhaitent cette année garder en Algérie, seront remplacés par des petits colons européens par priorité rapatriés $^{82}$ ". Cependant, en août 1962, plus d'un mois après l'indépendance, 65 enfants de Kerrata viennent en colonie de vacances à Laval, la Mayenne tenant à honorer son engagement envers son partenaire ${ }^{83}$.

Les relations établies entre des villes et des départements de l'Ouest et des communes et arrondissements d'Algérie ont-elles eu un impact sur la manière dont populations de l'Ouest envisageaient la guerre d'Algérie? Quel télescopage dans les journaux entre les annonces des évolutions de la politique algérienne, les rubriques telles que " Nos appelés en Algérie ",

80. Arch. dép. de Maine-et-Loire, 303 W 376, procès-verbal de la réunion de la commission de jumelage du 26 janvier 1962; lettres du préfet au président du conseil général des 16 et 25 juin 1962; lettre de Guy de Boischevalier, conseiller général, au préfet, 30 juin 1962.

81. Arch. dép. de la Vendée, 1494 W 659, télégramme du préfet du 23 mai et lettre du sous-préfet du 29 juin 1962.

82. Arch. dép. de Maine-et-Loire, 303 W 376, instructions du ministre des Affaires algériennes et du secrétaire d'État aux Rapatriés, juillet 1962.

83. Un seul article de presse rapporte ce séjour : "Ils sont arrivés de Kerrata (Algérie) ", Ouest-France, 9 août 1962; FoucAult, Rémy, " Mayenne-Kerrata (1959-1962)... ", art. cit., p. 14-15. 
les articles sur les colonies de vacances et les pages entières consacrées aux enfants du pays morts à la guerre. L'organisation et le déroulement des jumelages et des séjours d'enfants montrent un grand décalage entre la guerre psychologique - dont ressortent évidemment les jumelages et les colonies - qui est menée en Algérie et l'humanisme qui est à l'œuvre, tout simplement, en métropole à l'échelle locale. Il est à noter que la dimension humanitaire et la question du développement, à une époque où la sensibilisation aux problèmes du tiers-monde est de plus en plus prégnante ont sans doute contribué à faire agir les associations et les personnes.

Il faut insister sur le fait que ces parrainages/jumelages ont surtout été le fait d'initiatives personnelles pour les villes, d'une opération quasi imposée d'en haut pour les départements. Plus ou moins contraints, ils ont été de courte durée, et n'ont pas permis un ancrage durable des relations. Celles-ci ont surtout été humanitaires et à sens unique. La coupure de 1962 est nette, malgré quelques exceptions très limitées dans le temps, telle Château-Renault (Indre-et-Loire) qui accueille des harkis de la commune d'Arris avec laquelle elle est jumelée ${ }^{84}$. Dans certains cas, des monuments célébrant la présence française en Algérie sont transportés des villes algériennes vers les villes françaises jumelées et deviennent des lieux de mémoire. C'est le cas à Lyon pour le monument aux morts d'Oran élevé en 1927, installé à La Duchère en $1968^{85}$.

Le plus souvent, les jumelages sont très rapidement et complètement abandonnés. Le jumelage avec Barika est immédiatement oublié en Maineet-Loire; il ne reste aucune trace d'une quelconque relation qui se serait poursuivie. En 2015 et 2016, un appel à souvenirs, lancé sur le site des archives départementales et dans une revue d'histoire locale, ne donne aucun résultat. En Mayenne, même s'il était encore actif en 1962, le jumelage avec Kerrata est abandonné au profit d'un autre à l'échelle européenne, avec la Souabe. Les politiques de jumelage des villes de l'Ouest ont tous été réorientées vers des jumelages européens. Angers se lance en 1963 dans le premier jumelage tripartite avec Osnabrück et Haarlem ${ }^{86}$.

Ce n'est qu'à partir des années 1980 que des relations officielles sont nouées de nouveau avec des villes d'Algérie, mais sans rapport avec les précédents. Dès 1982, Rennes se jumelle avec Sétif, alors que l'Ille-et-Vilaine était jumelée avec Tablat; Rezé est jumelée avec Aïn-Defla tandis que la Loire-Atlantique l'était avec Paul-Cazelles (Aïn Oussoura aujourd'hui); La Roche-sur-Yon est jumelée depuis 1989 avec Tizi Ouzou, dont l'arron-

84. JoRDI, Jean-Jacques, Hamoumou, Mohand, Les harkis, une mémoire enfouie, Paris, Autrement, 1999, p. 92.

85. BRANche, Raphaëlle, La guerre d'Algérie. Une histoire apaisée?..., op. cit., p. 17; ANDRÉ, Marc, "Quand Lyon parrainait Oran... ", art. cit.

86. GuYOMARD, Sylvain, " Le premier jumelage européen tripartite : Angers-HaarlemOsnabruck " et RichIR, Amélie, "Le jumelage Souabe-Mayenne : une dynamique locale au service de l'Europe ", dans DENÉCHÈRE, Yves, VInCENT, Marie-Bénédicte, Vivre et construire l'Europe à l'échelle territoriale de 1945 à nos jours, Berne, Peter Lang, 2010, p. 37-48 et 69-81. 
dissement était jumelé avec l'Isère en 1960; Brest, qui était jumelée avec Mostaganem pendant la guerre, l'est depuis 1995 avec Béjaïa, tandis que c'est Perpignan qui est jumelée avec Mostaganem depuis 2011, non sans polémique autour de l'inauguration d'un Centre de Documentation des Français d'Algérie... Les jumelages des années de guerre ont donc été une expérience éphémère qui a néanmoins constitué pour les Français un des éléments d'une relation complexe à l'Algérie.

\section{RÉSUMÉ}

Dans le contexte des " événements d'Algérie ", le gouvernement français lance en 1959 une grande opération de jumelage des départements métropolitains avec les arrondissements d'Algérie. Avec une forte propagande en faveur de l'œuvre sociale de la France vers les populations d'Algérie, il s'agit de développer et de créer des liens concrets de solidarité sur le plan humain. Les départements de l'Ouest de la France acceptent tous de se jumeler, non sans exprimer des critiques face à cette injonction de l'État. Certains choisissent d'orienter leur aide vers les camps de regroupement forcé, ce qui est une manière de détourner l'opération en mettant en avant le sort très pénible des civils déplacés par l'armée française. Dans les départements, associations et citoyens s'engagent dans la dimension humanitaire de ces relations qui se concrétisent par des collectes, des envois de colis et des correspondances, surtout au bénéfice des enfants des arrondissements jumelés. Au-delà de la dimension politique et des critiques, l'enfance miséreuse d'Algérie s'impose unanimement comme la principale cause à promouvoir, celle qui réunit les engagements humanistes, religieux et laïques. L'Ouest accueille des centaines d'enfants lors de colonies de vacances estivales qui deviennent les actions emblématiques des jumelages. Elles établissent des liens directs entre les populations des deux côtés de la Méditerranée, mais éphémères et dont il ne reste pratiquement plus aucune mémoire.

\section{ABSTRACT}

In the context of the "events in Algeria", in 1959 the French government launched a twinning programme between départements in mainland France and Algerian arrondissements. Accompanied by forceful propaganda in favour of French social work towards the Algerian people, it was about developing and creating concrete links of solidarity on human terms. The départements of western France all accepted the twinning, though not without expressing criticism of this State injunction. Some chose to direct their aid towards forced regroupment camps, a way of diverting the operation by highlighting the difficult fate of the civilians displaced by the French army. In the départements, organisations and citizens became active in the humanitarian dimension of these relationships, which materialised as collections, care packages and letters, especially for the children of twinned arrondissements. Beyond politics and criticism, the destitute children of Algeria unanimously became the main cause to promote, uniting humanitarian, religious and secular commitments. The West hosted hundreds of children in summer camps which became the symbolic actions of these twinnings. They established direct, though ephemeral, links between the peoples on either side of the Mediterranean, links of which practically no memory remains. 